\title{
Observations of solar system objects with GAIA
}

\section{Detection of NEOS}

\author{
F. Mignard ${ }^{\star}$ \\ Observatoire de la Côte d'Azur, CERGA, UMR CNRS 6527, av. Copernic, 06130 Grasse, France
}

Received 2 August 2001 / Accepted 25 June 2002

\begin{abstract}
This paper discusses the observability by GAIA of solar system objects belonging to the group of near-earth objects, an important issue in the scientific program whose relevance for GAIA has been questioned. A simulation based on the most up-to-date distribution of the orbital parameters has been conducted over five years to determine the probability that an object will be in the field of view of the instrument with an apparent brightness larger than the limiting magnitude. It is found (for an albedo of 0.1) that most objects with a diameter larger than $\sim 2 \mathrm{~km}$ will be observed at least five times by GAIA and much more often for larger bodies. The detection survey will be $50 \%$ complete for objects of diameter $\sim 1 \mathrm{~km}$, while smaller objects $(D<0.5 \mathrm{~km})$ will usually escape detection. Very interestingly, it is shown that most observations will take place at small angular distance from the Sun, a zone where the ground-based surveys are the most biased, allowing to extend the existing surveys of ATENS and to probe into the virtually unknown population of objects orbiting inside the Earth's orbit. The distribution of the along-scan velocity averages near $40 \mathrm{mas} / \mathrm{s}(145 \mathrm{arcsec} / \mathrm{h})$, permitting excellent detection during a transit over the focal plane of GAIA. GAIA scientists therefore will be in a good position to alert a ground-based follow-up program.
\end{abstract}

Key words. techniques: miscellaneous - minor planets, asteroids

\section{Introduction}

The GAIA mission is primarily dedicated to the exploration of the Milky Way with the probing in depth of many types of stars of any ages, chemical composition or masses, using astrometry, multi-wavelength photometry and spectroscopy. In the proposal, Solar system objects appear also at a high level in the science program, with an impact on virtually any category of minor planets. It was clear from the beginning that solar system observations are not mere by-products of the mission, but are rather true scientific objectives belonging fully to the initial proposal, given the impact expected in this field. Perryman (2000) has stimulated interest by showing the remarkable capabilities of the instrument to detect and survey the NEO population in a way complementary to the on-going ground-based surveys. Near-Earth-Objects (NEOS) are small bodies in the solar system (asteroids and short-period comets) with orbits that regularly bring them close to the Earth and which are capable some day of striking our planet. Sometimes the term NEO is also used loosely to include all comets (not just short-period ones) that cross the Earth's orbit.

In the GAIA document (Gaia Study Report 2001) it is stated that GAIA will provide a detailed census of these objects down to the 20th magnitude, or equivalently down to diameters of about $260-590 \mathrm{~m}$ at $1 \mathrm{AU}$. As rightly pointed out during the formal presentation of the project, this statement could be misinter-

\footnotetext{
* e-mail: francois.mignard@obs-azur.fr
}

preted as saying that all objects down that size will be detected and measured by GAIA and a clarification was provided. The ambiguity resulted from incomplete treatment during the phase of study of the ability to detect faint sources. To determine the true possibilities one must cross the orbital distribution of the expected NEO population with the scanning law, look for the real distribution of apparent magnitude and hence compute realistic detection rates.

This was the main goal of an extensive simulation whose results are summarized in this paper, with an analysis of the scientific output to be expected from GAIA in the NEO survey. The detailed results are given in an appendix ${ }^{1}$.

Only the problems raised by the detection of the NEos are addressed here, the case of the main belt being deferred to a subsequent paper. This is justified by the fact that the orbital properties of the two populations are markedly different resulting into different observational conditions.

\section{Observing with GAIA}

GAIA approved in October 2000 within the ESA science programme, aims to create an extraordinarily precise three dimensional map of about one billion stars throughout our Galaxy with a target astrometric accuracy of $10 \mu$ as ( $\sim 50$ prad $)$ for a

\footnotetext{
1 The Appendix is only available in electronic form at http://www. edpsciences.org
} 


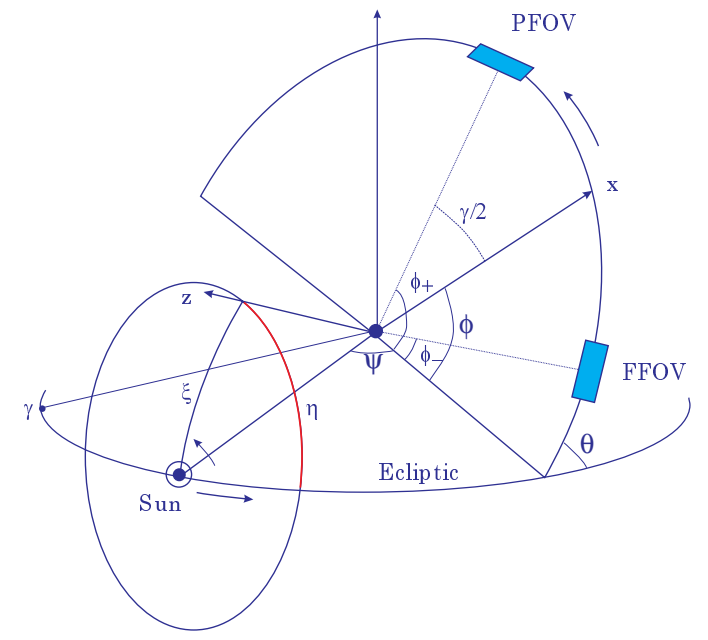

Fig. 1. The nominal scanning law of GAIA. The spacecraft rotates around the $z$-axis in 3 hours so that the Fovs swept approximately a circle on the sky. The $z$-axis is constrained to move on a sun-centered cone of 55 degrees with a period of 76 days, forcing the plane of scan to sway back and forth with inclinations between 35 to $145 \mathrm{deg}$. The axis of the cone follows the yearly solar motion.

star of 15th magnitude, yielding model-independent distances accurate to 10 per cent at several kiloparcecs, typically the distance of the globular clusters, the best tracers of the oldest stellar populations.

Within the scope of this paper there are few important features of the instrument and observational procedure that must be stressed:

- the limiting magnitude of detection is set at $G=20$, where $G$ is the magnitude scale defined through the bandpass of the CCD. It is very close to $V$ for solar spectra;

- there are three fields of view, each associated with a dedicated telescope and focal instrument. During the satellite rotation an object (as a rule) crosses the leading astrometric Fov in about $15 \mathrm{~s}$, then $\sim 1 \mathrm{~h}$ later, the trailing astrometric FOV and again after one hour the spectroscopic Fov;

- detection of a moving object can been performed in either Fov from its motion relative to the stars;

- the sky is scanned continuously, following a pre-defined pattern, with the satellite spin-axis kept at a fixed angle of $55^{\circ}$ from the Sun (Fig. 1). A precession motion of this axis over $\sim 70$ days allows a rather uniform scan of the full sky every six months. The scan period is three hours amounting to a displacement rate of the stellar images of $120 \mathrm{arcsec} / \mathrm{s}$.

A modified configuration is under study with a slower rotation, but this will not change the general conclusions of this paper.

\section{Orbital parameters of the NEOs}

\subsection{The model}

Near Earth Objects are small bodies orbiting the Sun with perihelion distances $q \leq 1.3$ AU and aphelion distances $Q \geq$ $0.983 \mathrm{AU}$, so that close encounters with the earth are possible. NEOs are subdivided into the three following sub-groups
(Rabinowitz et al. 1994), although several closely related definitions exist in the literature:

a - AMORs: Mars-crossing but not Earth-crossing asteroids with $1.0167<q \leq 1.3 \mathrm{AU}$

b - APOLLOS: include most Earth-crossing asteroids with $a \geq$ $1.0 \mathrm{AU}$ and $q \leq 1.0167 \mathrm{AU}$;

c - ATENS: asteroids with orbits largely inside the orbit of the Earth with $a<1.0 \mathrm{AU}$ and $Q \geq 0.983 \mathrm{AU}$.

Most NEOs are believed to be fragments of larger bodies of the main belt destroyed or eroded by internal collisions and that progressively drifted into mean-motion and secular resonances. Subsequently they enter into earth-approaching orbits from gravitational perturbations leading to transient a increase of the eccentricity (Morbidelli 1999).

In recent works, Bottke $(2000,2001)$ and collaborators have used several shortcuts to find the orbital and size distribution of near-earth asteroids. They integrate the orbits from the various sources from which the objects originate, to construct a steady-state model of the orbital and size distribution. Free parameters are fitted to the observations of a well characterized survey, namely Spacewatch, by assessing the bias effect between theoretical and observed distribution. The size distribution is assumed to be orbit-independent, which proves a very useful feature in the present work.

The five sources thought to provide most of the NEOs in the inner solar system are respectively (Bottke 2001):

1. the 3:1 mean-motion resonance with Jupiter for minor planets located at $\sim 2.5 \mathrm{Au}$;

2. asteroids in the $v_{6}$ secular resonance. This is a resonance between the aphelion or node motion of a minor planet with the 6 th eigenmode appearing in the secular evolution of the solar system. It is primarily due to Saturn;

3. asteroids on Mars-crossing orbits adjacent to the main belt; 4. the outer belt population of asteroids;

5. the comets belonging to the Jupiter family.

The probability distribution $R(a, e, i)$ is calculated for each mechanism by tracking 2-3 thousand test bodies and looking for large oscillations in eccentricity that can move the asteroid into the vicinity of the Earth. Each of the five mechanisms produces its own orbital distribution. The resulting distribution is a weighted combination of the five distributions.

The size distribution, or equivalently, the luminosity function in absolute magnitude (visual magnitude at $1 \mathrm{AU}$ from the Sun and from the Earth and with zero phase) is given by an independent random variable with a probability function given by,

$\mathrm{d} N=C \times 10^{\gamma(H-13)} \mathrm{d} H$

where $H$ is the absolute magnitude.

It is then easy to generate the random distribution between the brightest magnitude $\left(H_{\min }\right)$ and the faintest $\left(H_{\max }\right)$ with

$H=H_{\min }+\frac{1}{\beta} \log \left(1+\frac{\beta}{\alpha} *\right.$ rand $)$

where $\beta=\gamma \ln (10)=0.806$ and $\alpha=\beta /\left(\exp \left[\beta\left(H_{\max }-H_{\min }\right)\right]-\right.$ $1)$ and rand denotes a uniform random deviate on $[0-1]$. 
A simulation of a population of 20000 NEos was made available by A. Morbidelli from a Monte-Carlo simulation based on the above model. The number of objects was selected so as to produce reliable statistical results and to provide a significant population in each of the main sources of NEos. One must stress that this number is not meant to represent the expected size of the true population down to some limiting magnitude, a quantity largely unknown today. While the absolute number is arbitrary, the distribution over the orbital parameters and magnitude is expected to be realistic. Among the 20000 objects there are 1000 Atens, 12500 Apollos, 6000 Amors and about 500 outside these groups (usually with too small aphelion distances).

\subsection{The simulation}

The simulation is run over 5 years by using the GAIA nominal scanning law to determine the pointing directions (Fig. 1). At each time step the geocentric position and velocity vectors of the whole set of NEOs is computed. The distance is used to compute the apparent magnitude from the absolute magnitude and phase. The planet is considered as observable if $V<20$ at the time it appears in one of the fields of view. Several counters allow one to track each planet and to determine the number of observations, the typical distance (therefore magnitude) at which these observations take place, the solar elongation, the along- and cross-scan velocity, the phase angle, etc.

\section{The main results}

Most of the results with all the relevant plots are published electronically in an extended annex of this paper. In this section only a summary of the results is provided.

\subsection{Phases angles during the observations}

Intuitively the geometry of observations of NEos should lead to observations occurring predominantly at small phase angles, typically less than 30 degrees, since randomly selected observations take place at large distances from the Sun or from the Earth. However, in these configurations the planets are faint and hard to detect. So with a fixed limiting magnitude around $V=20$, just a fraction of all the field crossings will lead to a measurement. The resulting distribution of the phases shown in the annex is rather flat between 20 and 120 degrees, with no enhancement at small angle. So, due to the presence of large phase angles, the location of the photocentre will be significantly shifted from the center of mass and a correction will have to be applied to each observation, at least for the largest bodies.

\subsection{Solar elongations}

The direction of the Sun when a NEO is observed is a key parameter in the orbit coverage. From the ground it is known that observations are biased toward opposition, and that virtually no observations of NEOs can be made inside the orbit of the Earth, that is to say during day time. GAIA allows us to observe without degradation of efficiency as close as 35 degrees from the Sun. However the coverage is not uniform in time and the resulting distribution can only be obtained from a simulation crossing the scanning law and the orbital motion of the planets. One finds that about half of the observations are packed within 10 degrees of the minimum angle, while the other half are almost uniformly distributed between 50 to 145 degrees, the maximum allowed by the scanning law.

\subsection{Number of observations}

An observation is counted as a new one when an object crosses one of the fields of view and when it is brighter than $V=20$. For a star one expects about 65 such observations. In the case of a planet, not only does the object change its position on the sky, but its apparent magnitude may become larger than $V=20$, resulting in a censoring of the potentially achievable observations. In the real population the faintest objects are also more numerous and the overall censoring is in fact very large for objects with a diameter less than $\approx 2 \mathrm{~km}$. The simulation indicates that the survey of objects as faint as $H=20$ will be very limited, while for $H=18.5$ (diameter $\approx 1 \mathrm{~km}$ ) about 50 percent of the planets will be detected at least two times during the GAIA mission.

\subsection{Apparent velocity}

The magnitude is the only relevant parameter for the detection of sources by GAIA whatever their nature, as long as they are sufficiently point-like. However to tell that a detected source is not a star but a solar system object one needs an additional criterion. The key factor will be the quick recognition of a motion relative to the background stars, that can be detected from the drift of the image compared to the nominal displacement expected for a star. With the current configuration of GAIA it is thought that motion as small as 5 mas s$^{-1}$ is large enough to allow a recognition of a moving object during the crossing of one field of view. Smaller displacements will be noticeable by comparing the position from the crossing of the preceding and following field or after one revolution period. In the simulation the orbital motion is computed at each crossing and projected along the scan and cross-scan directions. The velocity distribution is approximately Gaussian with zero mean and a standard deviation of $30 \mathrm{mass}^{-1}$ (along-scan) and $40 \mathrm{mas} \mathrm{s}^{-1}$ (crossscan). Therefore there is a very good prospect that observed NEOs will be quickly recognized as moving objects within hours of their detection. A ground based follow up could then be activated to keep track of these objects.

\section{Implications for the GAIA survey}

We can now draw a few conclusions on the efficiency of GAIA in detecting NEos. Table 1 gives the probability of failure (no observation) or of success as a function of the magnitude and of the number of observations achievable. The table shows that for an object of absolute magnitude $H=18$, the probability that it is never detected by GAIA is 0.4 . In one case out of four it will be detected over less than 10 crossings out of an average of 
Table 1. Probability of observing a NEO of a given absolute magnitude with GAIA. The columns gives the probability that repeated observations occur a certain number of times, from $n=0$ (never observed) to $n>25$. The figures refer to the spectroscopic field. A limiting magnitude $V=20$ has been adopted for the detection.

\begin{tabular}{ccccc}
\hline \hline$H$ & $\begin{array}{c}n=0 \\
\%\end{array}$ & $\begin{array}{c}1<n \leq 10 \\
\%\end{array}$ & $\begin{array}{c}10<n \leq 25 \\
\%\end{array}$ & $\begin{array}{c}n>25 \\
\%\end{array}$ \\
\hline 14 & 2 & 1 & 6 & 91 \\
15 & 5 & 4 & 15 & 76 \\
16 & 11 & 9 & 25 & 55 \\
17 & 19 & 18 & 30 & 33 \\
18 & 40 & 27 & 21 & 12 \\
19 & 73 & 21 & 4 & 2 \\
20 & 89 & 9 & 2 & 0 \\
21 & 96 & 3 & 1 & 0 \\
\hline
\end{tabular}

85 for the spectroscopic field (Fig. A.4). One sees that the GAIA survey becomes very limited for objects fainter than $\sim 19$ mag (Diameter $<0.5 \mathrm{~km}$ ), with 75 percent of the NEOs repeatedly crossing the field of view unnoticed. On the other hand the GaIA survey will be virtually complete for sources brighter than $H=$ 16 , that is to say larger than $\sim 2 \mathrm{~km}$ in diameter. One can be more specific in term of the actual number of objects, assuming the luminosity function of the NEOs is known. This function has been estimated by several groups (see Bottke et al. 2001 for a discussion) with a remarkable agreement down to the kilometer sized objects. The population of NEos brighter than $H$ (with $H<$ 22) should be close to

$N(H)=17.4 \times 10^{0.35(H-13)}$

where the differential distribution of Bottke et al. (2001) has been transformed into a cumulative function. This model and the probability of detection from Table 1 lead to the number of potential detections listed in Table 2. The total number of objects detectable $(n>0)$ is just above 2000 and only half of them will have a good coverage during the mission.

Given the small numbers involved and the sustained ground-based activities, one may raise the question of how many new discoveries will be left for GAIA by 2012? Over the last few years the discovery rate of the largest NEOS ( $H<18$, kilometer size objects) has steadily increased to reach $\sim 100$ in 2001. At the end of 2001 the population of these large NEos numbers 550, amounting to about one half of the expected total population for objects larger than $1 \mathrm{~km}$ (http://neo.jpl.nasa.gov/missions/stats.html).

Assuming that the discovery or rediscovery is analogous to a random drawing with repetition, with equal probability for each drawing within a bin of absolute magnitude, the number $n$ of drawings required to realize a sample $x \%$ complete of a population of size $r$ is given by $n=-r \ln (1-x / 100)$. This number of drawings $n$ combines without distinction the discovery of new objects and the repeated observations of objects already detected. Therefore, a survey within a bin of magnitude will be $95 \%$ completed, when on average each object will have been seen three times. Doubling the current rate over the next 10 years (200 catches per year) yield 2000 detections. If
Table 2. Number of detections of NEOS with GAIA per range of absolute magnitude. The second column gives the expected number of NEos according to the model of Bottke et al. (2001). The column $N=0$ gives the number of non-detected objects, followed by those detected over less than 10 crossings or more than 10 . The figures refer to the spectroscopic field. A limiting magnitude $V=20$ has been adopted for the detection.

\begin{tabular}{ccccc}
\hline \hline$H$ & $N_{\text {neos }}$ & $n=0$ & $1<n \leq 10$ & $n>10$ \\
\hline $14.5-15.5$ & 70 & 3 & 3 & 64 \\
$15.5-16.5$ & 160 & 18 & 15 & 127 \\
$16.5-17.5$ & 360 & 70 & 70 & 220 \\
$17.5-18.5$ & 810 & 320 & 220 & 270 \\
$18.5-19.5$ & 1800 & 1300 & 380 & 120 \\
$19.5-20.5$ & 4050 & 3600 & 360 & 90 \\
$20.5-21.5$ & 9000 & 8600 & 270 & 90 \\
Total & 16250 & 13951 & 1318 & 981 \\
\hline
\end{tabular}

we add the 500 known objects this gives about 2500 detections, some of the objects appearing two or three times. This yields a completeness of about 90 percent in 2010 for the discovery of the large NEOs.

Therefore, as far as the discovery of new objects is concerned, the contribution of GAIA will be marginal. If we go fainter, most of the objects detected by GAIA are likely to be new ones, but the level of completeness will be too low to have a real scientific impact.

Hence, the importance of the observations of NEOs by GAIA lies elsewhere: for the NEOS GAIA is unique thanks to:

- the capability of observing close to the sun, where groundbased surveys are biased. This not only extends the orbital coverage for eccentric orbits, but allows one to detect objects with $a(1+e)<1$, something difficult to achieve from the Earth;

- the application of a homogeneous method of observation and analysis to all solar system objects;

- the multi-color photometry for the whole sample of NEOS observed;

- the realization of an all-sky coverage, allowing one to find NEOS on atypical orbits;

- the accurate orbit determination. The a priori knowledge of orbital parameters, even approximate, will ease considerably the identification over successive crossings, increase the rate of observation and permit a very precise orbit determination, much better than those derived from groundbased astrometry. A precise knowledge of the orbital elements is a a key factor to investigate the origin and evolution of the NEOS.

\section{Conclusions}

The main results reached from the crossing of a realistic NEoś population with the scanning law of GAIA are as follows:

- The detection of objects smaller than $\sim 500 \mathrm{~m}$ in diameter, corresponding to an absolute magnitude $H=20$ will have a low success rate, as most of the time these objects will be fainter than the limiting magnitude expected for the GAIA detection chain. 
- Objects brighter than $H=18$, corresponding to a diameter of $\sim 1 \mathrm{~km}$ will be observed many times during the mission and a $50 \%$ survey can be reasonably anticipated for objects of that size or larger.

- The survey will be virtually complete at $H=16$, that is to say for a diameter of about $2 \mathrm{~km}$.

- Many observations will take place at small angular distances from the Sun, making the GAIA survey complementary to the ground-based observations.

- This feature will allow one to explore the virtually unknown world of asteroids circling the Sun constantly within the orbit of the Earth.

- The along-scan velocity will be typically $40 \pm 30 \mathrm{mas} \mathrm{s}^{-1}$ $\left(145 \pm 108 \operatorname{arcsec}^{-1}\right)$, allowing quick recognition of the motion during a FOv crossing.

Acknowledgements. A. Morbidelli is gratefully acknowledged for having prepared and supplied a simulated file of orbital parameters for the NEos. Comments from M. Perryman have been very useful during the preparation of this work.

\section{References}

Bottke, F., Jedicke, R., Morbidelli, A., Petit, J. M., \& Gladman, B. 2000, Science, 288, 2190

Bottke, W. F. A., Morbidelli, A. R., Jedicke, R., et al. 2001, Icarus, in press

Bowell, E., Hapke, B., et al. 1989, in Asteroids II, ed. R. P. Binzel, T. Gehrels, \& M. S. Matthews (Univ. of Arizona Press, Tucson), 524556

GAIA 2000, Composition, Formation and Evolution of the Galaxy, ESA-SCI $(2000) 4$

Jedicke, R. 1996, AJ, 111, 970

Mignard, F. 2001, Detection of Near Earth Objects wiht GAIA, SAG-FM-007

Morbidelli, A. 1999, Celest. Mech., 73, 39

Perryman, M. A. C. 2000, Near-Earth Objects Observable with GAIA, SAG-MP-002

Perryman, M. A. C., et al. 2001, A\&A, 369, 339

Rabinowitz, D., Bowell, E., Shoemaker, E. M., Muinonen, K. 1994, In Hazards due to comets and Asteroids, T. Geherls (Ed. Univ. of Arizona Press) 